\section{SAT0643 VALUE OF POWER DOPPLER ULTRASONOGRAPHY FOR PREDICTION OF TREATMENT FOR RHEUMATOID ARTHRITIS (RA) IN EARLY ARTHRITIS DURING THE FIRST 12 MONTS OF FOLLOW-UP}

L. Mayordomo ${ }^{1,1}$, M.L. Velloso ${ }^{1}$, C. Jurado ${ }^{2}$, P. González-Moreno ${ }^{3}$

C. Gomez-Cano ${ }^{1}$, J.L. Marenco ${ }^{1}$, C. Almeida ${ }^{4} .{ }^{1}$ Rheumatology Department;

${ }^{2}$ Radiology Dp, Hospital Universitario Valme; ${ }^{3}$ Rheumatology Department, HVM;

${ }^{4}$ Research and Statistics Dp, Hospital Universitario Valme, SEVILLA, Spain

Background: There is a short window of opportunity for early diagnosis and treatment of rheumatoid arthritis, that may be crucial for reaching remission and a low rate of radiographic progression. High resolution power doppler ultrasonography (PDUS) is helpful in early detection of synovitis and may help to select patients that will need early establishmet of treatment for RA.

Objectives: To study whether the presence of basal power doppler signal in patients with early arthritis in a clinical basis treatment decision could predict the risk of receive treatment for $\mathrm{AR}$ at 3 and 12 months of follow-up.

Methods: We studied the presence of ultrasonographic Power Doppler (PD) signal on 28 joints (shoulders, elbows, wrists, MCPs, knees) and 44 joints (28 joints and in addition hips, Tarsus, ankles and MTPs), with a mid-range equipment GE L5, in 70 patients with suspected early arthritis. The patients were included with at least one of the following inclusion criteria: a) Swelling in 2 or more joints b) pain in MCPs, MTPs and/or the wrists c) morning stiffness of more than 30 minutes with $<12$ months duration of the symptoms. Clinical treatment decision was not awared to basal power doppler results. Statistical study: Chi-square, Fisher exact test, $p$ univariant and Odds Ratio calculation.

Results: The presence of basal power doppler signal in $\geq 1$ joints of 44 (PD44) at baseline shows statistically significant association with treatment with oral steroids $(p<0.001$, OR $8,6(2,4-30,5)$, DMARD p 0.028, OR 4,2 $(1,3-13,3)$, metotrexato (MTX) p 0.002 OR 6,6 $(1,9-22,1)$ but not sulfasalazine (SSZ) (p 0.145) at 3 months and with steroids $(p<0.0005$ OR $14,8(3,9-44,4)$, DMARDs $(p<0.0005$, OR 10,3 $(2,6-40,4)$, MTX $(p<0.0005$, OR 8,67 $(82,6-29,2))$, sulfasalazine $(p=0.001$, OR $8,7(2,2-33,9)$, at 12 months The presence of at least one joint with power doppler signal of 28 joints (PD28) was significantly associated to esteroids $(p=0.003$, OR $5,4(1,8-16,8)$ and metotrexate $(p=0.016$, OR $4,1(1,4-12,7)$ treatments in the first 3 months but not to other DMARDs $p=0.146$ (including sulfasalazine $p=0.416$ ) and with esteroid ( $p=0.01$ OR 7,3 (2.2-24,9), DMARD, $(p=0.03$, OR 5,8 $(1,8-18,7)$, MTX $(p=0.012$, OR 4,3 $(1,4-12,8)$, and sulfasalazine $(p=0.008$, OR 4,9 $(1,5-15,6)$ during first 12 months of follow-up. Predictive value of other risk factors for RA (RF, basal erosions=EROS BAS) is shown in the table below.

\begin{tabular}{|c|c|c|c|c|c|}
\hline $\begin{array}{l}\text { BASAL } \\
\mathrm{N}=70\end{array}$ & & $\begin{array}{l}\text { ESTEROID 12M } \\
\text { P univariate } \\
\text { OR IC 95\% }\end{array}$ & $\begin{array}{l}\text { DMARD 12M } \\
\text { P univariate } \\
\text { OR IC 95\% }\end{array}$ & $\begin{array}{l}\text { MTX 12M } \\
\text { P univariate } \\
\text { OR IC } 95 \%\end{array}$ & $\begin{array}{l}\text { SSZ 12M } \\
\text { P univariate } \\
\text { OR IC 95\% }\end{array}$ \\
\hline PD28 & $\begin{array}{l}\text { SI } \\
\text { NO }\end{array}$ & $\begin{array}{c}44 / 50(88 \%) \\
10 / 20(50 \%) \\
0,001 \\
\text { OR } 7,3(2,2-24,9)\end{array}$ & $\begin{array}{c}33 / 50(66 \%) \\
5 / 20(7,1 \%) \\
0,003 \\
\text { OR } 5,8(1,8-18,7)\end{array}$ & $\begin{array}{c}37 / 50(74 \%) \\
8 / 20(40 \%) \\
0,012 \\
\text { OR } 4,3(1,4-12,8)\end{array}$ & $\begin{array}{c}31 / 50(51,4 \%) \\
5 / 20(25 \%) \\
0,008 \\
\text { OR 4,9(1,5-15,6) }\end{array}$ \\
\hline PD44 & $\begin{array}{l}\text { SI } \\
\text { NO }\end{array}$ & $\begin{array}{c}47 / 52(90,4 \%) \\
7 / 18(38,9 \%) \\
<0,0005 \\
\text { OR } 14,8(3,9-44,4)\end{array}$ & $\begin{array}{c}35 / 52(67,3 \%) \\
3 / 18(16,7 \%) \\
<0,0005 \\
\text { OR } 10,3(2,6-40,4)\end{array}$ & $\begin{array}{c}40 / 52(76,9 \%) \\
5 / 18(27,8 \%) \\
<0,0005 \\
\text { OR } 8,67(2,6-29,2)\end{array}$ & \begin{tabular}{|c}
$33 / 52(63,5 \%)$ \\
$3 / 18(16,7 \%)$ \\
0,001 \\
OR 8,7(2,2-33,9)
\end{tabular} \\
\hline RCP & $\begin{array}{l}\text { SI } \\
\text { NO }\end{array}$ & $\begin{array}{c}40 / 45(88,9 \%) \\
14 / 25(56 \%) \\
0,003 \\
\text { OR } 6,3(1,9-21,3)\end{array}$ & $\begin{array}{c}\text { NS } \\
1,000\end{array}$ & $\begin{array}{c}37 / 45(82,2 \%) \\
8 / 25(32 \%) \\
P<0.0005 \\
\text { OR } 9,8(3,2-30,6)\end{array}$ & $\begin{array}{c}\text { NS } \\
0,624\end{array}$ \\
\hline FR & $\begin{array}{l}\text { SI } \\
\text { NO }\end{array}$ & $\begin{array}{c}19 / 20(95 \%) \\
35 / 50(70 \%) \\
0,028 \\
\text { OR 8,1(1-66,5) }\end{array}$ & $\begin{array}{l}\text { NS } \\
0,430\end{array}$ & $\begin{array}{c}19 / 20(95 \%) \\
26 / 50(52 \%) \\
0,001 \\
\text { OR } 17,5(2,1-141)\end{array}$ & $\begin{array}{c}\text { NS } \\
0,433\end{array}$ \\
\hline ACPA & $\begin{array}{l}\text { SI } \\
\text { NO }\end{array}$ & $\begin{array}{c}\text { NS } \\
0,272\end{array}$ & $\begin{array}{c}\text { NS } \\
0,193\end{array}$ & $\begin{array}{c}12 / 13(92,3 \%) \\
33 / 57(57,9 \%) \\
0,024 \\
\text { OR } 8,7(1,1-71,7)\end{array}$ & $\begin{array}{c}\text { NS } \\
0,622\end{array}$ \\
\hline EROS BAS & $\begin{array}{l}\text { SI } \\
\text { NO }\end{array}$ & $\begin{array}{c}\text { NS } \\
0,139\end{array}$ & $\begin{array}{l}\text { NS } \\
0,161\end{array}$ & $\begin{array}{c}26 / 30(86,7 \%) \\
17 / 35(48,6 \%) \\
0,002 \\
\text { OR 6,9(1,9-23,9) }\end{array}$ & $\begin{array}{c}\text { NS } \\
0,622\end{array}$ \\
\hline
\end{tabular}

Conclusions: Power doppler ultrasonography in at least one joint of 44 (PD44) may predict the patients that will need esteroids, any DMARD, MTX and SSZ teraphy in early arthritis at 3 and 12 months of follow-up. PD28 may predict the patients that will need esteroids, any DMARD, MTX and SSZ teraphy in early arthritis at 12 months, but may only predict the need of esteroid and MTX at three months.

Disclosure of Interest: None declared

DOI: 10.1136/annrheumdis-2017-eular.6910

\section{SAT0644 IS ANKLE INVOLVEMENT IMPORTANT IN RHEUMATOID ARTHRITIS?}

L. Enache, C. Mogosan, C. Popescu, G. Gutoiu, C. Codreanu. Rheumatology, Clinical Center of Rheumatic Disease, Bucharest, Romania

Background: The ankles may be affected by rheumatoid arthritis (RA). In daily evaluation of RA patients, the ankles are often neglected by clinical examinations, while composite activity scores do not include them

Objectives: The aim of the study is to assess the inflammatory lesions in RA ankles, regarding frequency and possible correlation with activity disease

Methods: The study included 76 RA patients fulfilling the 2010 ACR/EULAR classification criteria. In every patient we recorded clinical ankle involvement (ankle swelling and/or tenderness on physical examination); both ankles were evaluated by gray scale (GS) and Power Doppler (PD) ultrasound examination, graded from $0-3$. In each joint we assessed: tibio-talar joint (TT); tibialis anterior (TA) tendon; extensor hallux (EH) and extensor digitorum (ED) tendons; talonavicular joint (TN); tibialis posterior (TP) tendon; flexor digitorum (FD) and flexor hallux $(\mathrm{FH})$ tendons; peroneus brevis $(\mathrm{PB})$ and longus $(\mathrm{PL})$ tendons; Achilles tendon (AT); subtalar joints (SJ; posterior, lateral, medial recesses); plantar fascia $(\mathrm{PL})$. We recorded the following abnormalities: synovitis, tenosynovitis, bursitis, entesopathy and rupture. Activity scores (DAS28, DAS44, SDAI), inflammatory markers (ESR, CRP), serology (FR, ACPA) and HAQ were also assessed

Results: The mean age was $57.2 \pm 14.2$ years; $88 \%$ were women; mean disease duration was $12.2 \pm 9.2$ years; $56.6 \%$ had symptomatic ankles; $81.6 \%$ had ultrasound ankle abnormalities. The most frequent ultrasound pathology encountered was ST synovitis $(56.6 \%)$, followed by TT synovitis $(48.7 \%)$, TN synovitis $(38.2 \%)$, TP tenosynovitis (30.3\%), PL tenosynovitis (15.8\%). Ultrasound abnormalities were recorded in $93 \%$ of the symptomatic ankle patients and in $66.7 \%$ of the asymptomatic ankle patients $(p=0.008)$. Overall, $43 \%$ had positive PD signals, but the prevalence of positive PD signals was significantly higher in symptomatic ankle patients than in asymptomatic ankle patients $(60.5 \%$ compared to $21.2 \%, p=0.011)$. Statistically significant correlations were found between ultrasound inflammatory pathology (synovitis and tenosynovitis), activity disease markers and quality of life (Table 1)

Table 1. Correlations of GS ultrasound measures with disease activity and HAQ

\begin{tabular}{|c|c|c|c|c|c|c|c|}
\hline & ESR & CRP & DAS28 ${ }_{\text {CRP }}$ & DAS28 $8_{E S R}$ & DAS44 & SDAI & $\mathrm{HAQ}$ \\
\hline TT & 0.104 & $0,360^{\star \star}$ & $0,395^{\star \star \star}$ & $0,283^{*}$ & $0,317^{\star \star}$ & $0,352^{\star \star}$ & 0.150 \\
\hline TN & 0.159 & $0,426^{\star \star \star}$ & $0,442^{\star \star \star}$ & $0,330^{\star \star}$ & $0,366^{\star \star}$ & $0,391^{\star \star \star}$ & 0.007 \\
\hline PL & $0.272^{*}$ & $0.366^{\star \star}$ & $0.368^{\star \star}$ & $0.347^{\star *}$ & $0.362^{\star \star}$ & $0.340^{\star *}$ & $0,292^{*}$ \\
\hline PB & 0.074 & 0.139 & 0.200 & 0.215 & $0.231^{*}$ & 0.223 & $0,243^{*}$ \\
\hline TP & $0.408^{\star \star \star}$ & $0.466^{\star \star \star}$ & $0.543^{\star \star \star}$ & $0.548^{\star \star \star}$ & $0.558^{\star \star \star}$ & $0.542^{\star \star \star}$ & $0,268^{*}$ \\
\hline FD & 0.166 & 0.145 & $0.254^{*}$ & $0.260^{*}$ & $0.285^{\star}$ & $0.259^{\star}$ & $0,256^{*}$ \\
\hline
\end{tabular}

Note: the reported values represent the rho coefficients of bivariate Spearman correlations: ${ }^{*} \mathrm{p}<0,05 ;{ }^{* \star} \mathrm{p}<0,01 ;{ }^{* \star *} \mathrm{p}<0,001$.

Conclusions: Musculoskeletal ultrasound detected a high prevalence of inflammatory abnormalities in rheumatoid arthritis ankles, in both symptomatic and asymptomatic patients, while power Doppler signal was present mostly in symptomatic patients. The ultrasound pathology was highly correlated with activity disease markers and quality of life

References:

[1] M Backhaus, G-R Burmester, T Gerber et al., Guidelines for musculoskeletal ultrasound in rheumatology, Ann Rheum Dis 2001,; 60:641-649.

[2] M Alsuwaidi, B Ehrenstein, M Fleck et al., Ankle Joint in Rheumatoid Arthritis. An Ultrasound Study Using High Resolution- and Clour Doppler Ultrasound 2013 ACR/ARHP Annual Meeting, Abstract No 1969.

Disclosure of Interest: None declared

DOI: 10.1136/annrheumdis-2017-eular.6217

\section{SAT0645 MAGNETIC RESONANCE IMAGING AND HISTOPATHOLOGICAL CORRELATION IN DERMATOMYOSITIS}

$\underline{\text { M.V. Collado }}^{1}$, J.C. Milisenda ${ }^{2}$, X. Tomás ${ }^{3}$, A.I. García ${ }^{3}$, M. Faruch ${ }^{4}$, J.M. Grau ${ }^{2}$ on behalf of Muscle Research Unit, Internal Medicine Service, Hospital Clínic de Barcelona, Universidad de Barcelona and CIBERER. ${ }^{1}$ Reumatology, Instituto de Investigaciones Médicas Alfredo Lanari (UBA), Buenos Aires, Argentina;

${ }^{2}$ Internal Medicine; ${ }^{3}$ Radiology, Hospital Clínic de Barcelona, Barcelona, Spain;

${ }^{4}$ Radiology, CHU Toulouse Purpan, Toulouse, France

Background: Dermatomyositis represents one of the major forms among the inflammatory myopathies (IIM). Although the muscle biopsy remains the definitive test, MRI has been used to detect unique patterns of muscle involvement. To date, no studies have compared MRI with muscle pathology in naïve DM.

Objectives: To compare the pattern of muscle MRI with muscle pathology.

Methods: All the patients enrolled in the Hospital Clínic de Barcelona (HCB) from January 2009 to December 2016 with an available MRI, performed just before muscle biopsy were included. The HCB ethics committee approved this study, and written informed consent was obtained from each participant.

MRI data and the clinical and demographic features were prospectively collected. Patients were classified as having definite DM (ref 4). MRI was performed on a 1.5T with standardized protocol of whole body or scapular and pelvic girdle MRI, which include coronal and axial T1-weighted and STIR images. Image analysis was performed by two experienced musculoskeletal radiologists masked 